The AstrophysiCAL Journal, 508:844-853, 1998 December 1

(C) 1998. The American Astronomical Society. All rights reserved. Printed in U.S.A.

\title{
SYSTEMATICS OF RR LYRAE STATISTICAL PARALLAX. III. APPARENT MAGNITUDES AND EXTINCTIONS
}

\author{
ANDREW GOULD ${ }^{1}$ AND PIOTR PoPOWSKI ${ }^{2}$ \\ Ohio State University, Department of Astronomy, Columbus, OH 43210; gould@astronomy.ohio-state.edu, \\ popowski@astronomy.ohio-state.edu \\ Received 1998 May 18; accepted 1998 July 8
}

\begin{abstract}
We sing the praises of the central limit theorem. Having previously removed all other possible causes of significant systematic error in the statistical-parallax determination of RR Lyrae absolute magnitudes, we investigate systematic errors from two final sources of input data: apparent magnitudes and extinctions. We find corrections due to each of about $0.05 \mathrm{mag}$, i.e., about half the statistical error. However, these are of opposite sign and so approximately cancel out. The apparent-magnitude system that we previously adopted from Layden et al. was calibrated to the photoelectric photometry of Clube \& Dawe. Using Hipparcos photometry and archival modern ground-based photometry, we show that the Clube \& Dawe system is about 0.06 mag too bright. Extinctions were previously based on the map of Burstein \& Heiles, which was constructed from $\mathrm{H}$ I maps. We argue that extinctions should rather be estimated using the new map of Schlegel, Finkbeiner, \& Davis based on $C O B E$ and IRAS measurements of dust emission. This substitution increases the mean estimated extinction by about $0.05 \mathrm{mag}$, primarily because of a difference in the zero point of the two maps. Our final estimate for the absolute magnitude is $M_{V}=$ $0.77 \pm 0.13$ at $[\mathrm{Fe} / \mathrm{H}]=-1.60$ for a pure sample of 147 halo $\mathrm{RR}$ Lyrae stars, or $M_{V}=0.80 \pm 0.11$ at $[\mathrm{Fe} / \mathrm{H}]=-1.71$ if we incorporate kinematic information from 716 nonkinematically selected non-RR Lyrae stars from Beers \& Sommer-Larsen. These are 2 and $3 \sigma$ fainter than recent determinations of $M_{V}$ based on main-sequence fitting of clusters using Hipparcos measurements of subdwarfs by Reid and Gratton et al. Since statistical parallax is being cleared of systematic errors and since the probability of a more than $2 \sigma$ statistical fluctuation is less than $1 / 20$, we conclude that these brighter determinations may be in error. In the course of these three papers, we have corrected six systematic errors whose absolute values total $0.20 \mathrm{mag}$. Had these, contrary to the expectation of the central limit theorem, all lined up one way, they could have resolved the conflict in favor of the brighter determinations. In fact, the net change was only 0.06 mag.
\end{abstract}

Subject headings: dust, extinction - methods: statistical — stars: distances — stars: kinematics stars: variables: other (RR Lyrae)

\section{INTRODUCTION}

Statistical parallax appears to be an extremely robust method for measuring the absolute magnitude of halo RR Lyrae stars. Nevertheless, the results of this method are in serious conflict with several other determinations. In Paper II of this series (Popowski \& Gould 1998b, hereafter Paper II) we found

$M_{V}=0.74 \pm 0.12$, at $\langle[\mathrm{Fe} / \mathrm{H}]\rangle=-1.60$ (pure RR Lyrae),

for a sample of 165 halo RR Lyrae stars with high-quality proper motions from the Hipparcos (European Space Agency 1997) and Lick NPM1 (Klemola, Hanson, \& Jones 1993) surveys. We also combined this result with a separate determination based on a nonkinematically selected sample of 103 RR Lyrae stars and 724 non-RR Lyrae stars from Beers \& Sommer-Larsen (1995) and (taking account of the 0.45 correlation coefficient between the two samples) found

$M_{V}=0.77 \pm 0.10$, at $\langle[\mathrm{Fe} / \mathrm{H}]\rangle=-1.71$ (combined) .

The former value can be compared with measurements based on main-sequence fitting of globular clusters to subdwarfs with Hipparcos parallaxes that yield $M_{V} \sim 0.44$ \pm 0.08 (Reid 1997) or $M_{V} \sim 0.49 \pm 0.04$ (Gratton et al. 1997; Gratton 1998) at the same metallicity. (These com-

\footnotetext{
${ }^{1}$ Alfred P. Sloan Foundation Fellow.

${ }^{2}$ Ohio State University Presidential Fellow.
}

parisons take account of differences in the metallicity scales used by different authors as we discuss more fully in the Appendix.) If equation (1) is combined with the measurement of the dereddened apparent magnitude of RR Lyrae stars in the Large Magellanic Cloud (LMC) of $V_{0}=18.98$ \pm 0.05 (Hazen \& Nemec 1992; Popowski \& Gould 1998a, hereafter Paper I), this yields a distance modulus $\mu_{\text {LMC }}=$ $18.24 \pm 0.14$. (Here we have assumed an LMC metallicity $[\mathrm{Fe} / \mathrm{H}]=-1.8$, and a slope $M_{V}=$ const $+0.15[\mathrm{Fe} / \mathrm{H}]$, but the exact value of the slope makes very little difference because the metallicities in eq. [1] and of the LMC are so similar.) This result is quite low compared to the "traditional" value $\mu_{\mathrm{LMC}}=18.50$ and is even lower compared to those derived using Hipparcos-based calibrations of RR Lyrae stars and Cepheids: $\mu_{\mathrm{LMC}}=18.65 \pm 0.1$ (Reid 1997), $\mu_{\mathrm{LMC}}=18.63 \pm 0.06$ (Gratton et al. 1997), and $\mu_{\text {LMC }}=18.70 \pm 0.10($ Feast $\&$ Catchpole 1997$)$.

In principle, these discrepancies could be due to a greater than $2 \sigma$ statistical fluctuation. However, for Gaussian statistics, the probability of a $2 \sigma$ fluctuation is less than $1 / 20$. (Moreover, for the statistical-parallax determination, we have checked that the distribution of errors has Gaussian tails, even when the input data are not Gaussian distributed.) The usual cause of more than $2 \sigma$ discrepancies is not statistical fluctuations but systematic errors, and one is therefore led to suspect that there are unrecognized systematic errors in one or several of these measurements. Moreover, the conflict with equation (2) is even stronger, about 
$3 \sigma$. Although there are some additional assumptions that go into equation (2) that make it overall less robust than equation (1), the combined determination nevertheless argues against a large statistical fluctuation as the source of the discrepancy.

This is the third and final paper in a series designed to essentially eliminate the possibility of a significant systematic error in the statistical-parallax determination. Statistical parallax works in effect by forcing equality between the velocity ellipsoids as determined from radial velocities and from proper motions. That is, one can measure the nine parameters describing the velocity ellipsoid (three components of bulk motion $w_{i}$ plus six independent components of the velocity-dispersion tensor $C_{i j}$ ) from radial velocities alone. On the other hand, if one assumes some arbitrary absolute magnitude for the RR Lyrae stars, then one can infer their distances from their measured apparent magnitudes and estimated extinctions. The distances and proper motions yield the transverse velocities, and from these one can again estimate the nine parameters of the velocity ellipsoid. One could then adjust the assumed absolute magnitude so that the velocity ellipsoid from proper motions matched the velocity ellipsoid from radial velocities as closely as possible. In practice, one fits for all 10 parameters (nine for the velocities plus the absolute magnitude) simultaneously using maximum likelihood.

Logically, there are three possible ways for systematic errors to enter the determination. First, the mathematics of the method itself could introduce biases. Second, the RR Lyrae sample could fail to satisfy some of the physical properties assumed by the method. Third, one or more of the four major observational inputs (proper motions, radial velocities, apparent magnitudes, and extinctions) could be systematically in error. (A fifth observational input, metallicities, requires a separate discussion. Different studies may be on systematically different metallicity scales, and care must therefore be exercised when comparing the results from these investigations. See the Appendix.)

In Paper I, we investigated possible systematic errors arising from the mathematical method and physical assumptions. An example of a potential mathematical problem is that the likelihood method explicitly assumes that the velocity distribution is Gaussian, whereas, as we showed, the actual distribution is highly non-Gaussian. An example of a potential physical problem is that the method implicitly assumes that the velocity-dispersion tensor does not depend on location despite the fact that the stars are found at distances $(\lesssim 2 \mathrm{kpc}$ ) that are a significant fraction of the Galactocentric distance $\left(R_{0} \sim 8 \mathrm{kpc}\right)$. We examined a large number of such effects, some by vigorous Monte Carlo simulations and some with the aid of mathematical arguments. We corrected for all of them although most were smaller than $0.01 \mathrm{mag}$ and tended to mutually cancel one another. The largest correction ( 0.03 mag fainter) was due to Malmquist bias, which had been previously recognized but not previously incorporated into the analysis.

In Paper II, we investigated systematic errors arising from the first two observational inputs, proper motions and radial velocities. The proper motions are of greater concern because they are intrinsically more difficult to measure and hence have larger fractional errors. We had already noted in Paper I that if the proper-motion errors are misestimated, this can introduce significant systematic errors even if the proper motion themselves are unbiased. We used the precise Hipparcos proper motions (when available) to test the two large catalogs, Lick and Wan, Mao, \& Ji (1980, hereafter WMJ), that had previously been used and found that indeed the Lick errors had been slightly underestimated and the WMJ errors had been seriously underestimated. These two corrections moved $M_{V}$ brighter by 0.04 mag, but this was mostly compensated by random changes induced by substituting the more precise Hipparcos proper motions (when available) for the previous values. We also tested all three catalogs to search for nonstatistical errors and removed five questionable stars.

Radial velocities are in principle much easier to measure than proper motions. However, for pulsating variables, the measured velocity of (the atmosphere of) the star can differ from its center of mass by $\sim 50 \mathrm{~km} \mathrm{~s}^{-1}$ and hence an accurate velocity determination requires many measurements and/or good phasing. The quality of the radial-velocity data varies from star to star, and it was therefore possible that the errors had been either systematically overestimated or underestimated. In Paper II, we checked the entire system of the radial-velocity measurements by, in effect, determining the radial-velocity ellipsoid from the Beers \& SommerLarsen (1995) nonkinematically selected sample of metal-poor non-RR Lyrae halo stars. The resulting $M_{V}$ was consistent with the one derived from the pure RR Lyrae sample, indicating that the radial velocities are not a source of significant systematic error.

In brief, Paper I checked for and removed all sources of systematic error coming from the mathematics of the method and the physical assumptions about the sample, down to a level well below the statistical error. Paper II did the same for two of the observational inputs: proper motions and radial velocities.

Here we turn our attention to the remaining two observational inputs: apparent magnitudes and extinctions. At first sight, it does not seem that there could be much controversy about the apparent magnitude of $V \sim 12$ stars. However, exactly because the stars are bright, many were measured long ago. Layden et al. (1996) compiled photometric measurements from several sources and attempted to put them on a common system aligned with their large subsample from Clube \& Dawe (1980), which has photoelectric photometry and which they assumed to be equivalent to the modern (Landolt 1992) system. In particular, they found the photoelectric photometry of Bookmeyer et al. (1977) to be on average 0.06 mag fainter than that of Clube \& Dawe (1980) and transformed it accordingly (see Layden et al. 1996, Table 1). Thus, there are uncertainties in the apparent-magnitude scale of order $0.06 \mathrm{mag}$ that, according to equation (1), are half the size of the statistical error. In § 3, we test the Layden et al. (1996) system against Hipparcos photometry. We show that for $V \lesssim 12$, the untransformed Bookmeyer et al. (1977) photometry is in good agreement with Hipparcos. The Clube \& Dawe (1980) photoelectric photometry also agrees well with Hipparcos for $V \lesssim 10.5$ but is systematically brighter than Hipparcos by $\sim 0.06 \mathrm{mag}$ for $11 \lesssim V \lesssim 12$. The most straightforward interpretation of these results is that the Bookmeyer et al. (1977) photometry is more reliable than the Clube \& Dawe (1980) photometry and that therefore the Layden et al. (1996) system is too bright by about $0.06 \mathrm{mag}$. This conclusion is confirmed by the good agreement between Hipparcos and the high-quality photometry of Jones et al. (1992), Schmidt (1991), and Schmidt, Chab, \& Reiswig (1995). 
Extinctions pose another set of problems. For stars that are far from the Galactic plane, one can assume that they are above essentially all of the dust along their line of sight. One can therefore adopt the extinctions as measured for extragalactic objects along the same (or very nearby) lines of sight. Burstein \& Heiles (1982, hereafter BH) have constructed a map of such extinctions over a large fraction of the sky by combining galaxy counts and $\mathrm{H}$ I measurements. The extinction estimates of Layden et al. (1996) are based primarily on this map for the great majority of the sample. However, there are some lines of sight (particularly at low latitudes) for which $\mathrm{BH}$ do not give extinctions and others where the star is relatively close to the plane so that some of the dust may lie behind the star. In the latter cases, the BH map would overestimate the extinctions. For these stars, Layden et al. (1996) adopted other methods to estimate the extinction, notably the colors of the stars. Since the intrinsic color of RR Lyrae stars is a function of the period with relatively little scatter, this method should work well at least on average. However, Sturch (1966) had earlier used colors to estimate the extinctions toward a large sample of RR Lyrae stars, and Strugnell, Reid, \& Murray (1986) found that these estimates were systematically higher than their BH-based values by 0.11 mag. Layden et al. (1996) argued that the BH-based system was correct and attempted to put stars without $\mathrm{BH}$ extinctions on the same $\mathrm{BH}$ system. Nevertheless, it is important to note that if the Sturch (1966) system were correct, the dereddened apparent magnitudes would be systematically brighter by $0.11 \mathrm{mag}$, and hence the absolute-magnitude estimate would be brighter by the same amount. This would move the statistical-parallax estimates of $M_{V}$ and $\mu_{\mathrm{LMC}}$ closer by $1 \sigma$ to the estimates obtained using competing methods, and thus would help significantly to resolve the controversy.

In $\S 4$, we therefore reevaluate the extinctions using a different approach. First, we base our determinations on the new extinction map of Schlegel, Finkbeiner, \& Davis (1998, hereafter SFD). We argue that the SFD map is superior to the BH map both in its level of detail and in its zero point. (The zero point of SFD is about 0.06 mag higher in $A_{V}$ than the BH map.) Second, we restrict attention to stars that are more than $300 \mathrm{pc}$ from the Galactic plane. These lie beyond most of the dust and therefore the SFD extinctions require only small corrections. Third, we exclude the four stars with SFD extinctions $A_{V}>0.56$ since comparison with Layden et al. (1996) shows a systematic deviation for these stars and we are unable to determine which system is in error. We find a correction due to revised extinctions, which makes $M_{V}$ about 0.05 mag brighter. Combining the corrections due to revised apparent magnitudes and extinctions, we find that equations (1) and (2) are each increased (made fainter) by $0.03 \mathrm{mag}$. These changes are in fact mainly due to random fluctuations caused by the fact that we are using a slightly different sample (147 vs. 165 stars). The two systematic effects that we identify here almost precisely cancel out. Our results are all presented in $\S 5$, and we discuss the implications of these results in $\S 6$. We begin in $\S 2$ by describing our basic sample.

\section{SAMPLE}

As in Paper II, our initial sample of RR $a b$ Lyrae stars comes from two sources. First, Layden et al. (1996) give proper motions, radial velocities, apparent magnitudes, extinctions, and metallicities for 213 stars. Second, Hip- parcos gives proper motions for many of these 213 and for 19 additional stars for which there are radial velocities, apparent magnitudes, extinctions, and metallicities in Layden (1994). These are VX Scl, SX For, RX Col, HH Pup, RV Oct, TY Aps, XZ Aps, RW Tra, WY Pav, MS Ara, IN Ara, V455 Oph, V413 CrA, BK Dra, BN Pav, BP Pav, Z Mic, RY Oct, and SS Oct. In Paper II, we also included in our master file another star, BX Dra, which is probably an eclipsing variable, not an RR Lyrae star (Fernley et al. 1998). However, this was classified as a disk star and so did not enter the final sample. In Paper II, we eliminated a number of specific stars because of doubts about the quality of their proper motions. These included all stars with only WMJ proper motions. That is, our sample was composed entirely of stars with Lick and/or Hipparcos proper motions. We repeat this procedure in the current paper.

As in Paper II, we consider two different samples. First, we obtain a pure RR Lyrae sample of stars belonging to "halo-3" as defined by Layden et al. (1996). Second, we select a nonkinematic sample of both RR Lyrae stars and non-RR Lyrae stars using a metallicity criterion, $[\mathrm{Fe} / \mathrm{H}]<-1.5$. The latter are drawn from the sample of 1836 stars of Beers \& Sommer-Larsen (1995). As we discuss in $\S \S 3$ and 4, we slightly modify the selection procedures of Paper II to account for new apparent magnitude and extinction information.

\section{APPARENT MAGNITUDES}

Equations (1) and (2) were derived making use of photometry compiled by Layden et al. (1996, hereafter L96) when available and in a few cases from Layden (1994, hereafter L94) (which ultimately has the same sources). Of the 213 stars (including 162 "halo-3" stars) in L96, 57 (51 "halo-3") have photoelectric photometry from Clube \& Dawe (1980, hereafter CD80), 81 (54) have photoelectric photometry from Bookmeyer et al. (1977, hereafter B77), 7 (4) have Walraven photometry from Lub (1977), 21 (14) have CCD photometry from Schmidt (1991, hereafter S91) and Schmidt et al. (1995, hereafter S95), 8 (5) have preliminary photoelectric photometry from L96, and 39 (34) have photometry compiled by L94 from heterogeneous sources in the General Catalog of Variable Stars (Kholopov 1985, hereafter GCVS). L96 attempted to place all of these on a common system aligned with the photoelectric photometry of CD80.

In particular, they transformed the B77 photoelectric photometry and the S91 and S95 CCD photometry according to

$$
V_{\mathrm{L} 96}=0.146+0.983 V_{\mathrm{B} 77}
$$

and

$$
V_{\mathrm{L} 96}=-0.086+V_{\mathrm{S} 91, S 95} .
$$

Fernley et al. (1998) have fitted the light curves of 144 RR Lyrae stars (including 123 type $a b$ and 21 type $c$ ) using photometry data from Hipparcos. They transformed the Hipparcos $V_{H}$ magnitudes into Johnson $V$ magnitudes using $V=V_{H}-X$ where $X=0.09$ for type $a b$ and $X=0.06$ for type $c$, in accordance with the transformations given in the Hipparcos catalog at the appropriate colors for type $a b$ and type $c$, respectively. They tested these values against the precise ground-based measurements of Liu \& Janes (1990) for 13 stars with intensity-weighted means in the range $9.5 \lesssim V \lesssim 11.1$ including 11 type $a b$ and two type $c$. They found a mean offset of only $0.003 \mathrm{mag}$, with a scatter of 
0.007 mag. This gives high confidence both in the underlying Hipparcos data and in the procedure for recovering intensity-weighted means of Fernley et al. (1998, hereafter F98). We therefore propose to use the F98 data set to test the photometry of the larger L96 compilation.

Before doing so, however, we first note that the Hipparcos magnitudes require small additional corrections due to extinction. According to the Hipparcos catalog, in the neighborhood of $V-V_{H} \sim-0.09, d\left(V-V_{H}\right) / d(V-I)=$ -0.16, and therefore for reddened RR Lyrae stars one must further adjust the Hipparcos magnitudes by $-0.16 E(V-I) \sim-0.2 E(B-V)$. This results in our final estimate for Hipparcos-based Johnson $V$ magnitudes, $V_{h}$, for type $a b$ stars,

$$
\begin{aligned}
V_{h} & =V_{\mathrm{F} 98}-0.2 E(B-V) \\
& =V_{H}-0.09-0.2 E(B-V)(\mathrm{RRab}) .
\end{aligned}
$$

This change reduces the mean Hipparcos-based magnitudes by only $\sim 0.01 \mathrm{mag}$ relative to the F98 calibration.

We also follow F98 and remove three stars from our previous sample (as defined in Paper II). These are XZ Cet and AR Ser, which are anomalous Cepheids (not RR Lyrae stars), and BB Vir, which shows evidence of having a horizontal-branch star companion.

Figure $1 a$ shows the difference between the Hipparcosbased magnitudes $V_{h}$ and the magnitudes of stars drawn by L96 from two catalogs: B77 (triangles) and S91 + S95 (circles). Both catalogs have been transformed according to L96. The discrepancies are significant: $\left\langle V_{\mathrm{L} 96}-V_{h}\right\rangle=$ $-0.049 \pm 0.009$ for $\mathrm{B} 77$ and $\left\langle V_{\mathrm{L} 96}-V_{h}\right\rangle=-0.100$ \pm 0.011 for $\mathrm{S} 91+\mathrm{S} 95$, where the errors are standard errors of the mean. Figure $1 b$ shows the difference between the Hipparcos-based magnitudes and the original B77 and S91 + S95 magnitudes for the same stars. Here the agree- ment is excellent: $\left\langle V_{\mathrm{B} 77}-V_{h}\right\rangle=-0.006 \pm 0.009$ and $\left\langle V_{\mathrm{S} 91, \mathrm{~s} 95}-V_{h}\right\rangle=-0.014 \pm 0.011$. We conclude that the original B77 photometry was on the modern scale and that it was superior to the CD80 photometry. Less surprisingly, the S91 and S95 CCD photometry is also on the modern scale. We also show in Figure $1 b 16$ RR $a b$ Lyrae stars with high-quality photometry (squares) from Jones et al. (1992), a subset of which were used by F98 to test their Hipparcosbased magnitudes. The excellent agreement between Hipparcos and the solid points from Jones et al. (1992) and S91 + S95 over the range $9.6 \lesssim V \lesssim 12.3$ is strong evidence that the Hipparcos magnitude scale is correct over the full range of interest. (Note that Jones et al. 1992 actually consider 17 RR $a b$ Lyrae stars, but we are unable to reconstruct the undereddened photometry for one of these, SS Leo, from their sources, Liu \& Janes 1990 and Jones, Carney, \& Latham 1988.)

Based on these results, we therefore revise the catalog of apparent magnitudes as follows: We adopt the Hipparcosbased magnitudes of F98 (slightly adjusted for reddening as described by eq. [5]) whenever they are available. For the stars without Hipparcos-based magnitudes, we consider each of the six sources of photometry quoted by L96 separately. We adopt the original (untransformed) photometry of B77 and S91 + S95 since, from Figure $1 b$, these are in good agreement with Hipparcos. L96 reported preliminary photoelectric photometry for eight stars, of which one has a Hipparcos-based magnitude. For the remaining seven stars, we substitute the final values as reported by Layden (1997). Layden (1997) reports photoelectric photometry for one other star (V494 Sco) and a photoelectric recalibration of another star (V413 Oph) with photographic photometry previously transformed from L94 (see below). We adopt the Layden (1997) values in both cases. There are only three stars from Lub (1977) without Hipparcos-based magnitudes
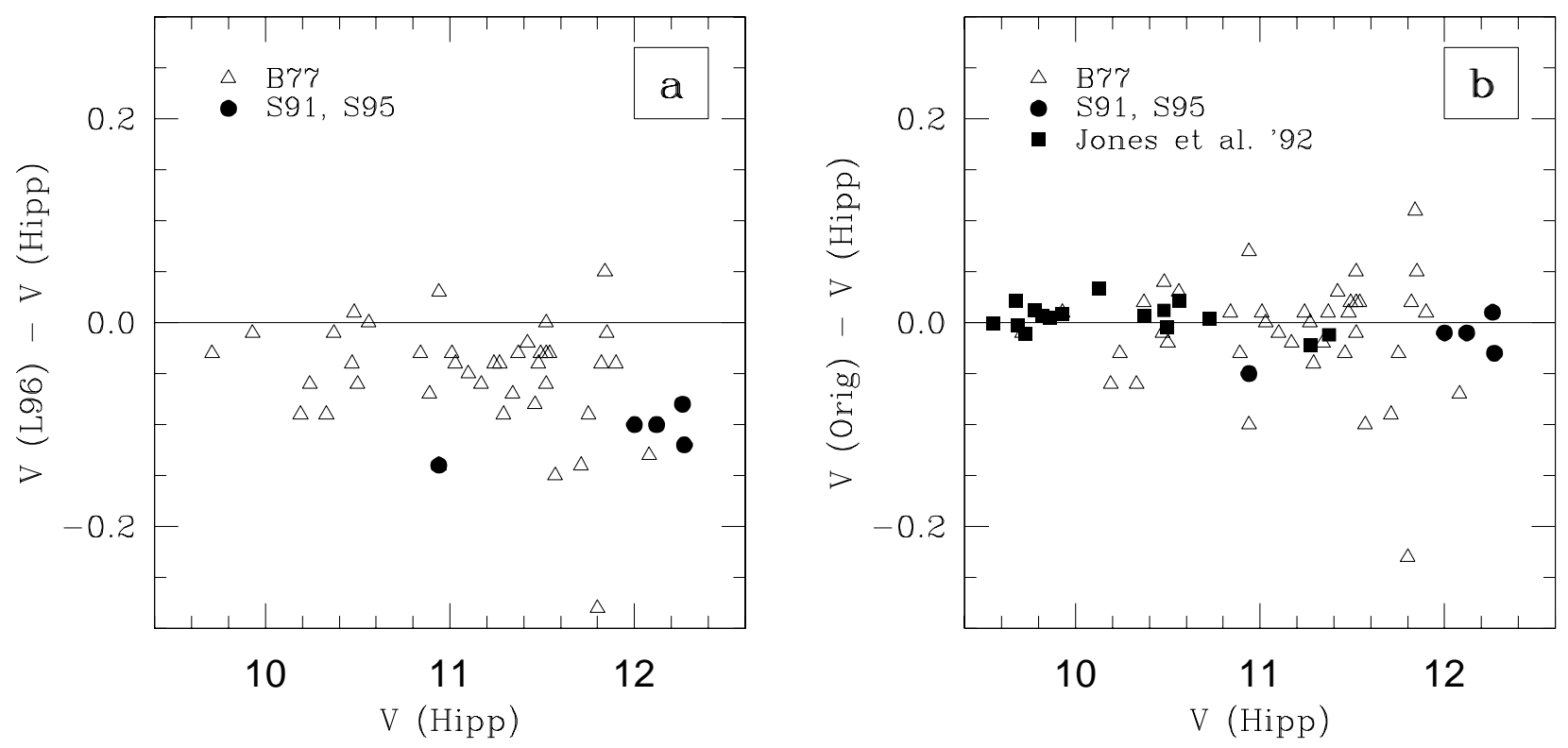

FIG. 1.-Difference between the $V$ magnitude of RR Lyrae stars as determined by F98 from Hipparcos data (slightly adjusted according toeq. [5]) and the $V$ magnitude for the same stars as determined by several other sources. Shown are stars with magnitudes reported by B77 (triangles), S91 + S95 (circles), and Jones et al. (1992) (squares). The last are shown in (b) only. In (a) we show the differences between the Hipparcos-based magnitudes and the magnitudes of B77 and S91 + S95 as transformed by L96 according to eqs. (3) and (4), respectively. There is a clear offset and trend with magnitude. In (b) we show the differences between the Hipparcos-based magnitudes and the magnitudes as originally reported by the various authors. Panel $(b)$ shows that the original magnitudes of all three samples are on the same system as Hipparcos. Moreover, the fact that the solid points (representing high-quality ground-based photometry) fall close to the zero line over the interval $9.6 \lesssim V \lesssim 12.3$ shows that F98's Hipparcos-based photometry is quite precise over the entire range of interest. 
(CP Aqr, AR Ser, and V494 Sco). AR Ser is an anomalous Cepheid (see above), and V494 Sco was remeasured by Layden (1997). CP Aqr is a disk star, and so does not enter into our final results. For completeness, however, we note that we derived a transformation $V=V_{\mathrm{L} 96} / 0.985-0.116$ for the Lub (1977) stars using the Hipparcos-based magnitudes of four of them. This leaves two sources: CD80 and L94.

Figure 2 shows $V_{\mathrm{CD} 80}-V_{h}$ as a function of $V_{\mathrm{CD} 80}$ (circles). For $V \lesssim 10.5, \mathrm{CD} 80$ is consistent with Hipparcos, but for $11 \lesssim V \lesssim 12, \mathrm{CD} 80$ is brighter, with a mean difference of $-0.06 \pm 0.01 \mathrm{mag}$. Thus, there is a clear trend with magnitude. In principle, it is possible that this trend is due to systematic errors in the Hipparcos-based photometry. However, as we discussed above, there is substantial evidence that Hipparcos is correct. Since Hipparcos agrees with B77, the same trend would appear in a comparison of CD80 and B77. Indeed, it was this trend that L96 measured to derive their "correction" for B77 given by equation (3). Since we established that B77 is correct and that the trend is due to systematic errors in CD80, it is appropriate to invert equation (3) to produce a correction for CD80,

$$
V=\frac{V_{\mathrm{CD} 80}-0.146}{0.983} \text {. }
$$

This is shown as a dashed line in Figure 2. Also shown are the $V_{\mathrm{CD} 80}$ magnitudes for the $18 \mathrm{CD} 80$ stars without Hipparcos-based magnitudes (crosses). For 13 of these CD80 stars, there is also B77 photometry. For these we plot the value of $V_{\mathrm{CD} 80}-V_{\mathrm{B} 77}$. Clearly, these differences follow

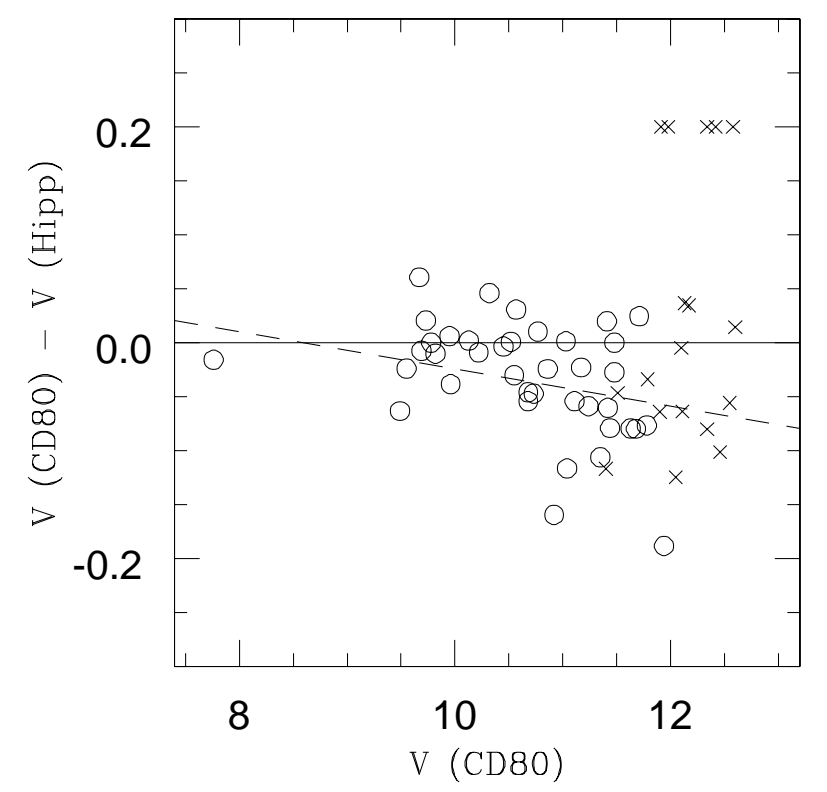

FIG. 2.-Difference between the $V$ magnitude of RR Lyrae stars as determined by F98 from Hipparcos data (slightly adjusted according to eq. [5]) and the $V$ magnitude for the same stars as determined by CD80 (circles). We plot this difference against $V(\mathrm{CD} 80)$ rather than $V$ (Hipp) to show apparent-magnitude distribution for 18 stars with photometry from CD80 but without Hipparcos photometry (crosses). For the 13 such stars for which photometry is also available from B77, the difference $V_{\mathrm{CD} 80}$ $-V_{\mathrm{B} 77}$ is shown. The remaining five stars have an ordinate of 0.2 . There is a clear trend with magnitude for the CD80 stars and this trend is similar for both the stars with Hipparcos photometry and with B77 photometry. The dashed line (eq. [6]) is the expected deviation of the CD80 stars from the true $V$ magnitude based on inverting equation (3) taken from L96, that is inverting L96's choice of the best available photometry and assuming it was B77 rather than CD80. the pattern of the Hipparcos-CD80 differences, confirming that B77 is on the Hipparcos system and CD80 is not. Therefore, for the five CD80 stars without B77 or Hipparcos photometry (shown with ordinate values of 0.2 ), we adopt the transformation given by equation (6). For the 13 CD80 stars with B77 photometry but without Hipparcos photometry, we adopt the average of the B77 photometry and the CD80 photometry as transformed by equation (6).

Figure 3 shows $V_{\mathrm{L} 96}-V_{h}$ for 27 stars that were drawn from GCVS by L94 and that have Hipparcos-based magnitudes. These include nine of the 39 such stars from L96 (the other 30 do not have Hipparcos-based magnitudes) plus 18 additional stars that were not analyzed by L96. (A 19th star, IN Ara, has a Hipparcos proper motion but no Hipparcos apparent magnitude because F98 found that the photometry was of too low a quality to extract a reliable result.) For these 18 stars, only L94 magnitudes were available. L96 do not specify exactly how they converted the L94 magnitudes, but by comparing the two catalogs, we find a very tight relation $V_{\mathrm{L} 96}=V_{\mathrm{L} 94}+0.0187\left(V_{\mathrm{L} 94}-10\right)$. Stars with photoelectric photometry are shown by crosses, and stars with photographic photometry are shown by circles. The six filled circles all derive from a single paper by Hoffmeister (1943) and are clearly grossly in error. These are all among the "18 additional stars" and so did not affect the L96 results but did affect the results of Paper II. Fortunately, none of the remaining 30 GCVS stars that lack Hipparcosbased magnitudes are drawn from this source, so it does not affect the present paper. Ignoring these six stars, the remaining 21 stars have $\left\langle V_{\mathrm{L} 96}-V_{h}\right\rangle=0.001 \pm 0.027$, with a scatter of $0.13 \mathrm{mag}$. This subsample is therefore overall of

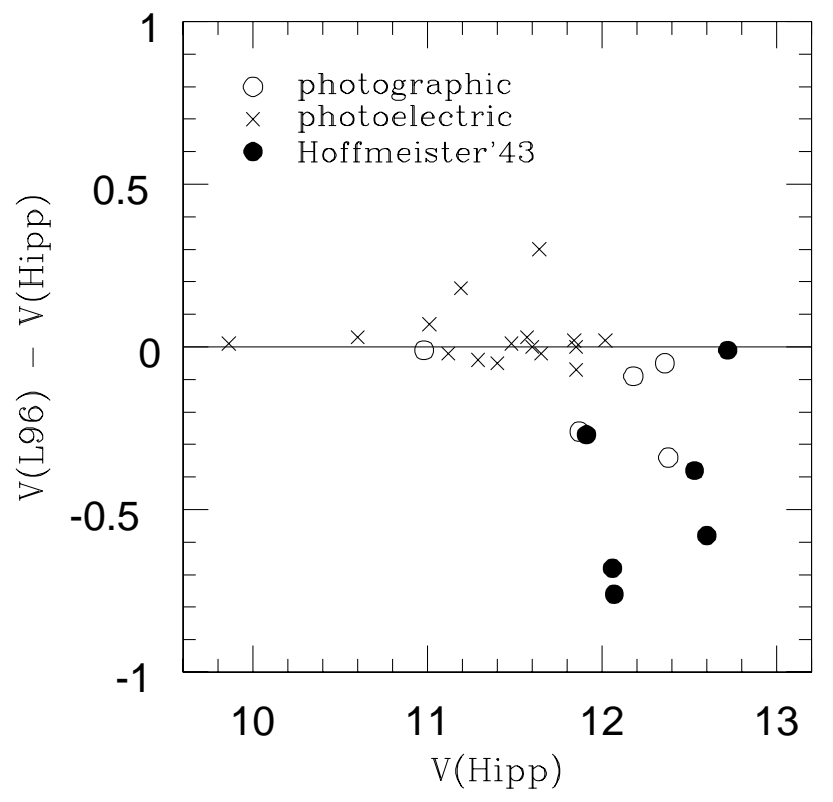

FIG. 3.-Difference between the $V$ magnitude of RR Lyrae stars as determined by F98 from Hipparcos data (slightly adjusted according to eq. [5]) and the $V$ magnitude for the same stars as determined by L96 by transforming photometry from GCVS. (For 18 of the stars only L94 photometry was available, but we have transformed these to the L96 system.) Shown are stars with photoelectric photometry (crosses) and photographic photometry (circles). The six solid circles are all from a single source (Hoffmeister 1943). If these are removed, the scatter is $0.13 \mathrm{mag}$, which is of lower quality than Hipparcos, B77, S91 + S95, and CD80 (adjusted according to eq. [6]), but is still acceptable. Of the 147 stars in the final sample, 24 have only GCVS photometry. 
TABLE 1

Photometry of 147 Halo Stars

\begin{tabular}{ccc}
\hline \hline Source & $\begin{array}{c}\text { Number of } \\
\text { Stars }\end{array}$ & Remarks \\
\hline F98 $\ldots \ldots \ldots \ldots \ldots \ldots \ldots \ldots$ & 72 & Based on Hipparcos \\
B77 $\ldots \ldots \ldots \ldots \ldots \ldots \ldots \ldots$ & 26 & \\
L96 $\ldots \ldots \ldots \ldots \ldots \ldots \ldots$ & 24 & Originally from GCVS \\
S91 + S95 $\ldots \ldots \ldots \ldots \ldots$ & 11 & \\
$($ B77 + CD80') $2 \ldots \ldots \ldots$ & 9 & CD80' from eq. (6) \\
Layden $1997 \ldots \ldots \ldots \ldots$ & 5 & \\
\hline
\end{tabular}

substantially lower quality than the rest of the sample. Given the fact that it comprises only $\sim 16 \%$ of the full final sample ( 24 out of 147 stars), and given that the mean difference is close to zero, the quality would still seem to be acceptable. However, Figure 3 has two additional, somewhat disturbing, features. First, the photographic measurements have substantially larger scatter. Second, the photographic measurements lie systematically below zero, whereas the photoelectric measurements lie systematically above zero. Thus, we approach the subset drawn from GCVS through L94 (and without Hipparcos-based magnitudes) cautiously. The primary results that we report incorporate these stars, but as a check, we also derive solutions by first eliminating them.

In the discussion above we described the general transformations (sometimes identities) that should be applied to existing photometric data and gave the number of stars in different categories referring to the whole master data set of 233 stars. In Table 1, we list sources of photometry and the number of stars with given photometry for our final sample of 147 halo stars.

\section{EXTINCTIONS}

Recent RR Lyrae statistical-parallax studies have all relied primarily on the $\mathrm{BH}$ reddening map to account for extinction (L96; Papers I and II; F98). We argue that this map should now be replaced by the SFD map for three reasons.

First, SFD is based on infrared emission (as measured by the $C O B E$ and $I R A S$ satellites), whereas $\mathrm{BH}$ is based on 21 $\mathrm{cm}$ measurements of neutral hydrogen. Infrared emission has a direct physical relation to the dust, whereas $\mathrm{H} \mathrm{I}$ is only indirectly related. Moreover, the $\mathrm{H}$ I method can underestimate the dust in dense regions either because the $21 \mathrm{~cm}$ line saturates or because $\mathrm{H} \mathrm{I}$ is converted into molecular hydrogen. SFD give an instructive example of regions that look very similar in $\mathrm{H} \mathrm{I}$, but have very different dust structures as measured by infrared emission.

Second, SFD covers more of the sky and does so in much greater detail. Therefore it is bound to replace $\mathrm{BH}$ for most applications, including work on the extragalactic distance scale. To obtain the most reliable results, one must measure the RR Lyrae absolute magnitude locally and the apparent magnitudes of RR Lyrae stars in external galaxies on the same system.

Third, the SFD map and the BH map differ systematically in the sense that SFD has 0.06 mag more extinction on average. Although there are arguments in favor of both zero points, the arguments for the SFD zero point appear more compelling to us. In any event, even if the SFD zero point were eventually proved wrong, such an error would, as noted in the previous paragraph, cancel out in most distance-scale applications. That is, the estimate of $M_{V}$ would be too bright, but the estimate of the dereddened apparent magnitude $V_{0}$ of RR Lyrae stars in external galaxies would be too bright by the same amount, so the distance modulus $\mu=V_{0}-M_{V}$ would be unaffected.

SFD establish the scale factor of their dust map by finding the slope and intercept of the linear fit to the plot of their dust measure against the observed $B-V$ color of elliptical galaxies. They determine the zero point from the correlation at high Galactic latitudes of their far-infrared dust emission measure with $\mathrm{H}$ I emission (after removing the zodiacal light using the correlation with $25 \mu \mathrm{m}$ emission). Once the slope and the zero point are fixed, every point on the sky is assigned an extinction. If the zero point of this relation were substantially in error, then the point with the lowest extinction would most likely be either below zero or substantially above zero. In fact, the extinction in the least obscured region averaged over a $1^{\circ}$ aperture, and so statistically highly significant, is positive, but quite close to zero, $A_{V}=0.01$. If the $\mathrm{BH}$ zero point were correct, then the SFD map should be adjusted downward by $0.06 \mathrm{mag}$, and the best estimate for the dust at this lowest point would be $A_{V} \sim-0.05$. Thus, both the method for determining the SFD zero point and the actual result appear very sound.

In order to use the SFD map, we must initially restrict attention to RR Lyrae stars that lie sufficiently far from the Galactic plane that most of the dust along the line of sight to extragalactic objects actually lies in front of the star. We choose a minimum distance from the plane of $z_{\min }=300 \mathrm{pc}$, and we assume that the dust has a scale height of $h=130$ pc. Thus, for a star at height $z=300 \mathrm{pc}$, we assume that $[1-\exp (-|z| / h)] \times 100 \%=90 \%$ of the dust lies in front of the star and hence that the reddening $E(B-V)$ is $90 \%$ of the value given by SFD. We adopt $R_{V}=A_{V} / E(B-V)=3.1$. Of course, it is possible that along any given line of sight all of the dust lies within $300 \mathrm{pc}$, and that along a few lines of sight, a significant patch of dust lies beyond $300 \mathrm{pc}$. However, from the point of view of measuring the absolute magnitude of RR Lyrae stars, all that is important is that this scale-height relation is correct on average.

Figure 4 shows L96 extinctions versus SFD-based extinctions for stars with $|z|>300$ pc. The SFD-based extinctions incorporate the correction for a dust scale height of $h=130 \mathrm{pc}$. The L96 extinctions are based primarily on the BH system, and, like our values, were originally corrected for a dust scale height. The diagonal line has a slope of 1 and an intercept of -0.05 to account for the zero point differences between SFD and BH as determined from RR Lyrae stars. The solid triangles are "halo-3" stars and the open triangles are disk stars. The latter are not included in our statistical-parallax solution but are useful for studying systematics in extinctions. For $A_{V} \lesssim 0.56$, the points are grouped closely around the line, whereas at higher values they tend to fall below the line. We do not know the cause of this deviation, whether the SFD-based extinctions are too high or the BH-based extinctions are too low. Plausible arguments could be made either way. In this paper we try to avoid all possible sources of systematics, and we therefore eliminate the four stars (circles) with SFD extinctions $A_{V}>$ 0.56 .

The restrictions $|z|>z_{\max }$ and $A_{V}<0.56$ remove only about $10 \%$ of the sample. Nevertheless, it would be nice to reincorporate these excluded stars, provided that there was a way to estimate their extinctions on the same scale as the 


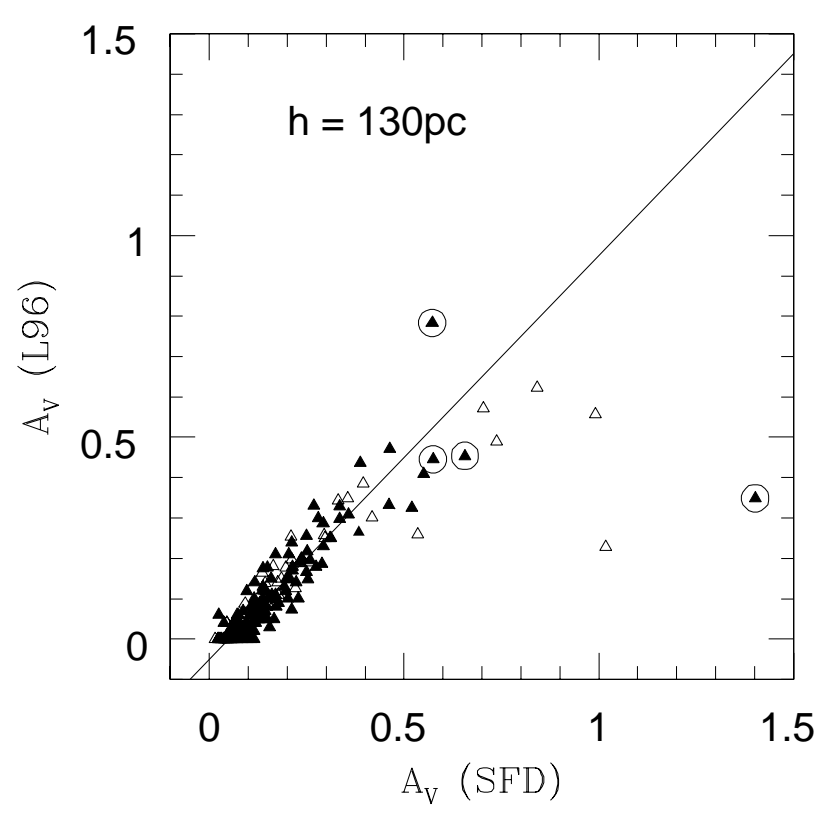

FIG. 4.-Extinctions of L96 (based primarily on BH) versus extinctions based on SFD (including a correction for a dust scale height of $h=130 \mathrm{pc}$ ). Shown are "halo-3" stars (solid triangles) and disk stars (open triangles). The diagonal line has a slope of 1 and an intercept of -0.05 , to account for the zero point difference between the two maps. The points systematically fall below the line for $A_{V} \gtrsim 0.56$. We therefore eliminate the four halo-3 stars with SFD extinctions $A_{V}>0.56$ (circles).

SFD map. Alcock et al. (1998a, 1998b) have measured the correlation between $\log$ period $P_{0}$ and intrinsic $(V-K)_{0}$ color based on SFD extinction estimates of a sample of 16 RR $a b$ Lyraes with well-measured light curves. They find

$$
(V-K)_{0}=1.052 \pm 0.013+(1.059 \pm 0.147)\left(\log P_{0}+0.29\right) \text {. }
$$

Unfortunately, of the 15 excluded stars in the "halo-3" statistical-parallax sample, we are able to find mean $K$ magnitudes of sufficiently high quality for this purpose for only one (TU Uma). We therefore use only stars with $|z|>300 \mathrm{pc}$ and $A_{V}<0.56$, which yields a sample of 147 stars of which 24 have photometry from GCVS. (MS Ara is above $300 \mathrm{pc}$ if its new Hipparcos apparent magnitude is adopted and below $300 \mathrm{pc}$ if its old GCVS magnitude is used. We include this star in all solutions to permit a fair comparison.)

\section{RESULTS}

Table 2 shows the statistical-parallax solutions for our final sample of 147 "halo-3" RR Lyrae stars (as defined in L96 and additionally specified in Paper II). This sample is smaller than the 165 stars that we analyzed in Paper II primarily because of the removal of stars with poorly determined SFD extinctions as discussed in $\S 4$, but also because of the removal of two anomalous Cepheids and one star with a possible horizontal-branch companion as discussed in $\S 3$. We show four solutions: (1) without any changes relative to the data used in Paper II, (2) with new apparent magnitudes but with extinctions taken from Paper II, (3) with new (SFD) extinctions but with apparent magnitudes taken from Paper II, and (4) with new extinctions and new apparent magnitudes. The numbers in parentheses are the errors. Note that whereas row (1) is not strictly comparable to the results of Paper II because of the different number of stars (147 vs. 165), the results are actually quite similar.

Column (2) is the distance-scaling factor $\eta$, which is normalized so that $\eta=1$ for the absolute-magnitude scale of L96,

$$
\eta \equiv 1 \Rightarrow M_{V}=0.95+0.15[\mathrm{Fe} / \mathrm{H}](\mathrm{L} 96) .
$$

Hence, the final result of incorporating the new apparent magnitudes and new extinctions for 147 halo RR Lyrae stars is

$$
M_{V}=0.77 \pm 0.13 \text {, at }\langle[\mathrm{Fe} / \mathrm{H}]\rangle=-1.60 \text { (pure RR Lyrae) }
$$

Columns (3), (4), and (5) give the bulk motion of the sample in $\mathrm{km} \mathrm{s}^{-1}$ relative to the local Galactic frame (with radial coordinate pointing outward). Here, the Sun is assumed to be moving relative to this frame at $v_{\odot}=(-9$, $232,7) \mathrm{km} \mathrm{s}^{-1}$. The bulk motion is $w_{i}=(6 \pm 14,15 \pm 13$, $1 \pm 8) \mathrm{km} \mathrm{s}^{-1}$. Columns (6), (7), and (8) give the square roots of the diagonal terms of the velocity-dispersion matrix, $C_{i j}^{1 / 2}$ in $\mathrm{km} \mathrm{s}^{-1}$. The final row reports $C_{i i}^{1 / 2}=\left(\sigma_{\pi}, \sigma_{\theta}\right.$, $\left.\sigma_{z}\right)=(171 \pm 11,99 \pm 8,90 \pm 7) \mathrm{km} \mathrm{s}^{-1}$. We do not display the off-diagonal terms of $C_{i j}$ as we did in Papers I and II because they are close to zero and uninteresting.

All the results in Table 2 have been corrected for Malmquist bias, scatter in the $M_{V}-[\mathrm{Fe} / \mathrm{H}]$ relation, bias due to the anisotropic distribution of program stars on the sky, the effects of the non-Gaussian RR Lyrae velocity distribution, and rotation from the heliocentric frame to the localGalactic frames of the stars, as discussed in detail in Paper I.

As we discussed in $\S 3,24$ of the 147 stars in this sample have photometry from GCVS, which is overall of lower quality (scatter $\sim 0.13 \mathrm{mag}$ ) than that of the other 123 stars. These stars should not be excluded on these grounds because this scatter is still small compared to the intrinsic scatter of the method, $N^{1 / 2} \sigma_{M_{V}}=1.57 \mathrm{mag}$, where $N=147$ is the size of the sample and $\sigma_{M_{V}}=0.13 \mathrm{mag}$ is the error in equation (9). (See Appendix A of Paper II for an additional discussion.) Nevertheless, for completeness we have determined the solution without these stars and find $M_{V}=$ $0.77 \pm 0.14$, almost exactly the same as equation (9).

TABLE 2

Comparison between Four Kinematically Selected RR Lyrae Samples

\begin{tabular}{cccccccc}
\hline \hline $\begin{array}{c}\text { Description } \\
(1)\end{array}$ & $\begin{array}{c}\eta \\
(2)\end{array}$ & $\begin{array}{c}w_{1} \\
(3)\end{array}$ & $\begin{array}{c}w_{2} \\
(4)\end{array}$ & $\begin{array}{c}w_{3} \\
(5)\end{array}$ & $\begin{array}{c}C_{11}^{1 / 2} \\
(6)\end{array}$ & $\begin{array}{c}C_{22}^{1 / 2} \\
(7)\end{array}$ & $\begin{array}{c}C_{33}^{1 / 2} \\
(8)\end{array}$ \\
\hline Old $V$, old $A_{V} \ldots \ldots$. & $0.982(0.058)$ & $4.4(14.5)$ & $15.5(12.4)$ & $0.7(7.9)$ & $172.1(10.8)$ & $98.2(8.0)$ & $89.6(7.2)$ \\
New $V$, old $A_{V} \ldots \ldots$. & $0.953(0.057)$ & $5.6(14.4)$ & $15.2(12.5)$ & $0.8(8.0)$ & $170.9(10.7)$ & $99.3(8.1)$ & $89.8(7.2)$ \\
Old $V$, new $A_{V} \ldots \ldots$. & $1.005(0.060)$ & $4.8(14.5)$ & $15.6(12.4)$ & $0.8(7.9)$ & $172.2(10.8)$ & $98.2(8.0)$ & $89.6(7.2)$ \\
New $V$, new $A_{V} \ldots \ldots$ & $0.975(0.058)$ & $5.9(14.4)$ & $15.4(12.5)$ & $1.0(8.0)$ & $171.1(10.7)$ & $99.3(8.1)$ & $89.8(7.2)$ \\
\hline
\end{tabular}

NoTE.-The bulk motion and velocity dispersions are given in $\mathrm{km} \mathrm{s}^{-1}$. Quantities in parentheses are the uncertainties in parameter determinations. 
In $\S 4$ we adopted $h=130 \mathrm{pc}$ for the exponential scale height of the dust. We find that if we instead use $h=100 \mathrm{pc}$ or $h=160 \mathrm{pc}$, equation (9) is changed by about $0.001 \mathrm{mag}$. Even if we were to use $h=300 \mathrm{pc}$, the change would be only $0.01 \mathrm{mag}$.

In Paper II, we also considered a nonkinematically selected sample of 827 stars with $[\mathrm{Fe} / \mathrm{H}] \leq-1.5$, including 103 RR Lyrae stars and 724 non-RR Lyrae stars from Beers \& Sommer-Larsen (1995). The available evidence suggests that RR Lyrae stars are similar in their kinematics to other metal-poor stars (Ryan \& Lambert 1995; Chiba \& Yoshii 1998), and that the kinematics of metal-poor stars are not a strong function of metallicity (Beers \& Sommer-Larsen 1995). Therefore the non-RR Lyrae stars should define the same velocity ellipsoid as the RR Lyrae stars, despite the fact that their mean metallicity is $\langle[\mathrm{Fe} / \mathrm{H}]\rangle \sim-2.2 \mathrm{com}$ pared to $\langle[\mathrm{Fe} / \mathrm{H}]\rangle \sim-1.8$ for the RR Lyrae subsample. Under this assumption, the large number of non-RR Lyrae stars can independently define the radial-velocity ellipsoid and so yield a check on the radial-velocity ellipsoid (and hence the statistical-parallax solution) defined by RR Lyrae stars alone. That is, only radial velocities of the non-RR Lyrae stars define the first velocity ellipsoid, and this is later matched to the distance-dependent velocity ellipsoid obtained using both radial velocities and proper motions of RR Lyrae stars. This allows one to determine the distancescale parameter $\eta$.

Here we repeat this procedure with a few changes. First, we eliminate all of the RR Lyrae stars that have questionable apparent magnitudes or extinctions as discussed in $\S 4$. Second, we slightly change the selection criterion from $[\mathrm{Fe} / \mathrm{H}] \leq-1.5$ to $[\mathrm{Fe} / \mathrm{H}]<-1.5$. The reason for this is that L96 assigned to seven RR Lyrae stars for which there were no measured metallicities, an assumed metallicity [Fe/ $\mathrm{H}] \equiv-1.5$, which was the mean for their entire sample. One may show that for the pure RR Lyrae samples of L96, Paper I, Paper II, and this paper, this assumption introduces an utterly negligible random error of $0.002 \mathrm{mag}$. Thus, the assumption is justified in order to maximize the size of the usable sample. However, incorporation of these stars could introduce a bias for the metallicity-selected sample we are now considering. By slightly changing the criterion to $[\mathrm{Fe} / \mathrm{H}]<-1.5$, we eliminate these stars and very few others. We then obtain a sample of 87 RR Lyrae stars with $\langle[\mathrm{Fe} / \mathrm{H}]\rangle=-1.81$ and 716 non-RR Lyrae stars with $\langle[\mathrm{Fe} / \mathrm{H}]\rangle=-2.22$. Table 3 gives the results for this sample. The description of the columns and rows is the same as for Table 2. The results correspond to

$$
M_{V}=0.82 \pm 0.13 \text {, at }\langle[\mathrm{Fe} / \mathrm{H}]\rangle=-1.81 \text { (nonkinematic), }
$$

$w_{i}=(4 \pm 9,34 \pm 9,2 \pm 5) \mathrm{km} \mathrm{s}^{-1}$, and $C_{i i}^{1 / 2}=\left(\sigma_{\pi}, \sigma_{\theta}\right.$, $\left.\sigma_{z}\right)=(160 \pm 7,109 \pm 8,94 \pm 5) \mathrm{km} \mathrm{s}^{-1}$. Following the procedure discussed in Paper II, we have added in quadrature a systematic error of $0.04 \mathrm{mag}$ in going from Table 3 to equation (10) to take account of possibly different levels of thick-disk contamination in the RR Lyrae and non-RR Lyrae solutions.

As in Paper II, we combine the two determinations (eqs. [9] and [10]) taking account of the 0.44 correlation coefficient between them (see Appendix B to Paper II) and obtain,

$$
M_{V}=0.80 \pm 0.11 \text {, at }\langle[\mathrm{Fe} / \mathrm{H}]\rangle=-1.71 \text { (combined) . }
$$

Note that equations (9) and (11) are in good agreement with equations (1) and (2).

\section{DISCUSSION}

We take equation (9) as the primary result of this paper because the statistical-parallax solution for the pure RR Lyrae sample requires essentially no additional assumptions. By contrast, the nonkinematic solution of equation (10) and, by implication, the combined solution of equation (11) require the additional assumption that metal-poor RR Lyrae stars have the same kinematics as metal-poor non-RR Lyrae stars. Although this assumption is not absolutely secure, there are a number of very strong arguments in its favor. First, as we discussed above and in Paper II, there is no evidence that RR Lyrae and non-RR Lyrae stars can be distinguished kinematically. Second, the available evidence suggests that kinematics are independent of metallicity for $[\mathrm{Fe} / \mathrm{H}]<-1.5$. Third, the pure RR Lyrae and nonkinematic solutions for $\eta$ agree within their errors, even taking account of the 0.44 correlation coefficient between them (see Tables 2 and 3). Fourth, there are no statistically significant differences between the individual velocity components of the solution in Table 2 for 147 RR Lyrae stars and the individual velocity components of the solution based only on the 716 metal-poor Beers \& Sommer-Larsen (1995) stars (i.e., as determined from radial velocities alone). The latter differs somewhat from the solution in Table 3 and is given by $w_{i}=(-2.2 \pm 9.6$, $38.3 \pm 11.0,1.1 \pm 5.5) \mathrm{km} \mathrm{s}^{-1}$ and $C_{i i}^{1 / 2}=(160.0 \pm 10.1$, $118.7 \pm 13.1,92.6 \pm 6.1) \mathrm{km} \mathrm{s}^{-1}$. Taking the difference between these six parameters and those in Table 2 and dividing by the errors yields $(0.5,-1.4,-0.2,0.8,-1.2$, $-0.3)$. The component $w_{2}$ differs the most for those two solutions, but this is expected because the pure RR Lyrae sample was selected by removing stars with prograde orbits, whereas the nonkinematic sample was, of course, selected without kinematic criteria. The component $C_{22}^{1 / 2}$, which has

TABLE 3

Comparison between Four Samples of Nonkinematically Selected Stars with $[\mathrm{Fe} / \mathrm{H}]<-1.5$

\begin{tabular}{cccccccc}
\hline \hline $\begin{array}{c}\text { Description } \\
(1)\end{array}$ & $\begin{array}{c}\eta \\
(2)\end{array}$ & $\begin{array}{c}w_{1} \\
(3)\end{array}$ & $\begin{array}{c}w_{2} \\
(4)\end{array}$ & $\begin{array}{c}w_{3} \\
(5)\end{array}$ & $\begin{array}{c}C_{11}^{1 / 2} \\
(6)\end{array}$ & $\begin{array}{c}C_{22}^{1 / 2} \\
(7)\end{array}$ & $\begin{array}{c}C_{33}^{1 / 2} \\
(8)\end{array}$ \\
\hline Old $V$, old $A_{V} \ldots \ldots$. & $0.936(0.051)$ & $3.8(8.5)$ & $34.4(8.7)$ & $2.1(4.8)$ & $160.0(6.6)$ & $109.0(8.3)$ & $94.4(5.0)$ \\
New $V$, old $A_{V} \ldots \ldots$. & $0.915(0.050)$ & $3.9(8.5)$ & $34.3(8.7)$ & $2.1(4.8)$ & $159.7(6.6)$ & $109.2(8.3)$ & $94.4(5.0)$ \\
Old $V$, new $A_{V} \ldots \ldots$. & $0.958(0.052)$ & $3.9(8.5)$ & $34.4(8.7)$ & $2.2(4.8)$ & $160.2(6.6)$ & $108.9(8.3)$ & $94.3(5.0)$ \\
New $V$, new $A_{V} \ldots \ldots$ & $0.937(0.051)$ & $4.0(8.5)$ & $34.3(8.7)$ & $2.1(4.8)$ & $159.9(6.6)$ & $109.1(8.3)$ & $94.4(5.0)$ \\
\hline
\end{tabular}

NoTE.-The bulk motion and velocity dispersions are given in $\mathrm{km} \mathrm{s}^{-1}$. Quantities in parentheses are the uncertainties in parameter determinations. 
the second largest difference, is also affected by selection criteria. Finally, if there were any systematic differences between the RR Lyrae stars and the non-RR Lyrae stars used in the nonkinematic sample, we would expect that it would be in the sense of the non-RR Lyrae stars having more extreme kinematics because they are on average more metal poor. This would drive the radial-velocity ellipsoid to higher dispersions and faster (relative to the Sun) bulk motion, and hence would cause one to overestimate distances (and luminosities) of the RR Lyrae stars when one attempted to match their proper motions to these high, non-RR Lyrae radial velocities. That is, the only plausible bias of this method is in the same direction as would be needed to resolve the discrepancy between statistical parallax and other methods of determining the absolute magnitude of RR Lyrae stars and opposite in sign from the actual difference between the nonkinematic and pure RR Lyrae samples. In brief, although equation (11) does not sit on as firm a foundation as equation (9), it does argue very strongly against the idea that equation (9) is the result of a large statistical fluctuation, particularly a fluctuation in the direction of underestimating the RR Lyrae luminosity.

As we discussed in the introduction, equation (9) is in conflict at the $2 \sigma$ level with the values determined from main-sequence fitting of clusters at the same metallicity of $M_{V} \sim 0.44 \pm 0.08$ (Reid 1997) or $M_{V} \sim 0.49 \pm 0.04$ (Gratton et al. 1997; Gratton 1998). There are only four possible explanations for such a discrepancy: (1) a rare $(<1 / 20)$ statistical fluctuation, (2) a substantial difference between cluster stars and field stars in the magnitude of the horizontal branch, (3) a systematic error in the mainsequence fitting distances to clusters, or (4) a systematic error in the statistical-parallax measurement.

In this series of three papers, we have eliminated explanation (4). Explanation (1) is of course always possible but is unlikely. Gratton (1998) has suggested explanation (2), that field and cluster horizontal branches might be different. However, two lines of evidence weigh against this possibility. First, as Gratton (1998) notes, comparison of the apparent magnitudes of RR Lyrae stars in LMC clusters with those of neighboring field RR Lyrae stars (for which the reddening should be quite similar) shows a mean offset of only $0.05 \pm 0.02$. However, this argument strictly applies only to LMC RR Lyrae stars: there still could be a difference between field and cluster RR Lyrae stars in the Galaxy, which, unlike the LMC, is a large spiral and probably has had quite a different formation history. In fact, Sweigert \& Catelan (1998) have produced models of two clusters with rising blue horizontal branches (NGC 6388 and NGC 6411) that have RR Lyrae stars several tenths of a magnitude brighter than those of canonical horizontal-branch scenarios. The models invoke nonstandard features, either high helium abundance, high rotation velocity, or helium mixing at the tip of the giant branch, which cause the stars to have longer periods at fixed temperature and metallicity. Such long periods are actually observed for the two known RR Lyrae stars in NGC 6388, enhancing the plausibility of this explanation. However, Catelan (1998) has shown that the period-temperature diagrams for RR Lyrae stars in five clusters that have been used for main-sequence fitting are actually quite similar in appearance to those of field stars of similar metallicity. Hence, although some cluster horizontal branches may be brighter than those of the field, this does not appear to be the case for the clusters with mainsequence fitting distances.

We therefore consider that explanations (1), (2), and (4) are all rather unlikely and that a systematic error in the main-sequence fitting distances is the most plausible explanation for the discrepancy. One possible cause of a systematic error in the main-sequence fitting distances is that the metallicities of the local subdwarfs might be on a different scale from those of the clusters (determined from giants). Specifically, if the subdwarf metallicities were too low (or the giant metallicities too high) then intrinsically brighter subdwarfs would be matched to the cluster main sequences, leading to an overestimate of the cluster distance and of the luminosity of its horizontal branch. Recently, King et al. (1998) have found intriguing evidence of a possible misalignment of this sort. They measured the metallicities of M92 subgiants (not quite subdwarfs, but with higher gravities than giants) and obtained metallicities up to $\frac{1}{2}$ dex lower than those of M92 giants. Although there are a number of possible explanations for this result, one is that the metallicities of giants are being systematically overestimated or those of subdwarfs are being systematically underestimated.

We would like to thank A. Layden for clarifying a number of points related to the database he and his collaborators compiled and for sending us a copy of the GCVS reference list that was missing from our library. This work was supported in part by grants AST 94-20764 and AST 97-27520 from the NSF.

\section{APPENDIX}

\section{METALLICITIES}

RR Lyrae luminosities are a function of metallicity, and therefore it is best if the mean metallicity of the calibrating stars be the same as that of the stars whose distance one wants to measure. Failing this, one should at least know the difference between the two metallicities and the slope of absolute magnitude with metallicity.

Metallicity itself (in the sense of abundance of Fe relative to the solar value) is not an observable. Rather it is a parameter derived from observables (line strengths, colors, etc.) on the basis of atmosphere models. In order for RR Lyrae stars to be effective distance indicators, it is not necessary that their true metallicity be known. Rather, as discussed above, it is only necessary to know the difference in metallicities between different samples. In principle, this difference could be determined directly from the observables. In practice, the observables are usually summarized as a single number: the "metallicity." Thus, as long as two metallicities are measured on the same "system" (and hence codify the observables in the same way) the role of RR Lyrae stars as distance indicators is not affected by absolute errors in the metallicity. Metallicity therefore enters the statistical-parallax calibration in a substantially different way from the other observational inputs, which is why we treat it separately. Of course, it is of some interest to know what the true metallicities of RR Lyrae stars are, and consequently what 
are their absolute magnitudes as a function of true metallicity. However, this question does not bear directly on the distance scale.

The L94 metallicities (which we have adopted) are pinned to the Zinn \& West (1984, hereafter ZW84) metallicity scale for globular clusters. (See, e.g., Fig. 1 of L94.) Hence, our statistical-parallax calibration has the ZW84 scale embedded in it. When this calibration is applied to other RR Lyrae stars with metallicities on the ZW84 scale (such as Reid 1997), one can make a direct comparison regardless of whether the ZW84 scale is actually correct. However, there are numerous other metallicity scales that can differ by several tenths of a dex from ZW84. For example, Jurcsik \& Kovács (1996) find that the L94 (i.e., ZW84) scale is related to the Suntzeff, Kraft, \& Kinman (1994) scale by $[\mathrm{Fe} / \mathrm{H}]_{S}=0.957[\mathrm{Fe} / \mathrm{H}]_{L}+0.200$. Comparing individual cluster metallicities of Gratton et al. (1997) with ZW84, we find that the former are about 0.1 dex more metal rich at $[\mathrm{Fe} / \mathrm{H}]_{\mathrm{Zw} 84} \sim-2$ and about 0.25 dex more metal rich at $[\mathrm{Fe} / \mathrm{H}]_{\mathrm{Zw} 84} \sim-1.5$. Hence, when we compared our absolutemagnitude calibration at $[\mathrm{Fe} / \mathrm{H}]_{\mathrm{zw} 84} \sim-1.6$ with Gratton et al. (1997) and Gratton (1998, specifically eq. [15]), we actually used their absolute magnitude at $[\mathrm{Fe} / \mathrm{H}]=-1.4$. Note that if we had made the comparison "naively" at the Gratton (1998) metallicity of $[\mathrm{Fe} / \mathrm{H}]=-1.6$, the discrepancy between the two methods would have been more severe by 0.04 mag. For this case the comparison is relatively straightforward. However, in general, metallicities are often quoted without making clear what scale they are on, so that it may be difficult to carry out rigorous comparisons in some cases. In any event, one should always proceed cautiously whenever making any such comparison.

For the comparison with Gratton (1998), the situation is made simpler by the fact that the Gratton (1998) slope of $S=d M_{V} / d[\mathrm{Fe} / \mathrm{H}]$ is well constrained. However, if we consider comparing the statistical-parallax result $\left(\right.$ at $[\mathrm{Fe} / \mathrm{H}]_{\mathrm{SP}}=$ -1.60 ) with the value obtained by another method at a different metallicity, $[\mathrm{Fe} / \mathrm{H}]_{*}$, then there is an additional uncertainty in the comparison, even if the two metallicities are put on the same scale. This uncertainty is $\Delta S \Delta[\mathrm{Fe} / \mathrm{H}]$, where $\Delta[\mathrm{Fe} / \mathrm{H}]=$ $[\mathrm{Fe} / \mathrm{H}]_{\mathrm{SP}}-[\mathrm{Fe} / \mathrm{H}]_{*}$ and $\Delta S \sim 0.15$ is the difference of plausible slopes reported in the literature, $S=0.15$ to $S=0.30$.

Alcock, C., et al. 1998a, ApJ, 494, 396 $1998 \mathrm{~b}$, in preparation

Beers, T. C., \& Sommer-Larsen, J. 1995, ApJS, 96, 175

Bookmeyer, B. B., Fitch, W. S., Lee, T. A., Wisniewski, W. Z., \& Johnson,

H. L. 1977, Rev. Mexicana Astron. Astrofis., 2, 235 (B77)

Burstein, D., \& Heiles, C. 1982, AJ, 87, 1165 (BH)

Catelan, M. 1998, ApJ, 495, L81

Chiba, M., \& Yoshii, Y. 1998, AJ, 115, 168

Clube, S. V. M., \& Dawe, J. A. 1980, MNRAS, 190, 591 (CD80)

European Space Agency. 1997, The Hipparcos Catalog (Paris: ESA) SP-1200

Feast, M. W., \& Catchpole, R. W. 1997, MNRAS, 286, L1

Fernley, J., Barnes, T. G., Skillen, I., Hawley, S. L., Hanley, C. J., Evans, D. W., Solano, E., \& Garrido, R. 1998, A\&A, 330, 515 (F98)

Gratton, R. G. 1998, MNRAS, 296, 739

Gratton, R. G., Fusi Pecci, F., Carretta, E., Clementini, G., Corsi, C. E., \& Lattanzi, M. G. 1997, ApJ, 491, 749

Hazen, M. L., \& Nemec, J. M. 1992, AJ, 104, 111

Hoffmeister, C. 1943, Kleinere Veröffentilichungen der Universitätssternwarte zu Berlin-Babelsberg, Vol. 27

Jones, R. V., Carney, B. W., \& Latham, D. W. 1988, ApJ, 326, 312

Jones, R. V., Carney, B. W., Storm, J., \& Latham, D. W. 1992, ApJ, 386, 646

Jurcsik, J., \& Kovács, G. 1996, A\&A, 312, 111

Kholopov, P. N. 1985, General Catalog of Variable Stars, (4th ed.; Moscow: Nauka)(GCVS)
REFERENCES

King, J. R., Stephens, A., Boesgaard, A. M., \& Deliyannis, C. P. 1998, AJ, 115,666

Klemola, A. R., Hanson, R. B., \& Jones, B. F. 1993, Lick Northern Proper Motion Program, NPM1 Catalog (Greenbelt: Astronomical Data Center), A1199

Landolt, A. U. 1992, AJ, 104, 340

Layden, A. C. 1994, AJ, 108, 1016 (L94) 1997, PASP, 486, 107

Layden, A. C., Hanson, R. B., Hawley, S. L., Klemola, A. R., \& Hanley, C. J. 1996, AJ, 112, 2110 (L96)

Liu, T., \& Janes, K. A. 1990, ApJ, 354, 273

Lub, J. 1977, Ph.D. thesis, Leiden Univ.

Popowski, P., \& Gould, A. 1998a, ApJ, 506, 259 (Paper I)

.1998b, ApJ, 506, 271 (Paper II)

Reid, I. N. 1997, AJ, 114, 161

Ryan, S. G., \& Lambert, D. L. 1995, AJ, 109, 2068

Schlegel, D. J., Finkbeiner, D. P., \& Davis, M. 1998, ApJ, 500, 525 (SFD)

Schmidt, E. G. 1991, AJ, 102, 1766 (S91)

Schmidt, E. G., Chab, J. R., \& Reiswig, D. E. 1995, AJ, 110, 2493 (S95)

Strugnell, P., Reid, N., \& Murray, C. A. 1986, MNRAS, 220, 413

Sturch, C. 1966, ApJ, 143, 774

Suntzeff, N. B., Kraft, R. P., \& Kinman, T. D. 1994, ApJS, 93, 271

Sweigert, A. V., \& Catelan, M. 1998, ApJ, 501, L63

Wan, L., Mao, Y. Q., \& Ji, D. S. 1980, Ann. Shanghai Obs., 2, 1

Zinn, R., \& West, M. J. 1984, ApJS, 55, 45 (ZW84) 